\title{
BRDOKH OSEN
}

NATIONAL LABORATORY

BNL-113265-2001-IR

\section{CSEWG 2016 Minutes}

\author{
D. Brown, M. Herman, A. Sonzogni, M. Chadwick, \\ A. Kahler, Y. Danon, D. Smith, M. Dunn, G. Nobre
}

November, 2016

National Nuclear Data Center

Brookhaven National Laboratory

\author{
U.S. Department of Energy \\ Office of Science, \\ Office of Nuclear Physics
}




\section{DISCLAIMER}

This report was prepared as an account of work sponsored by an agency of the United States Government. Neither the United States Government nor any agency thereof, nor any of their employees, nor any of their contractors, subcontractors, or their employees, makes any warranty, express or implied, or assumes any legal liability or responsibility for the accuracy, completeness, or any third party's use or the results of such use of any information, apparatus, product, or process disclosed, or represents that its use would not infringe privately owned rights. Reference herein to any specific commercial product, process, or service by trade name, trademark, manufacturer, or otherwise, does not necessarily constitute or imply its endorsement, recommendation, or favoring by the United States Government or any agency thereof or its contractors or subcontractors. The views and opinions of authors expressed herein do not necessarily state or reflect those of the United States Government or any agency thereof. 


\section{CSEWG 2016 Minutes}

\section{Table of Contents}

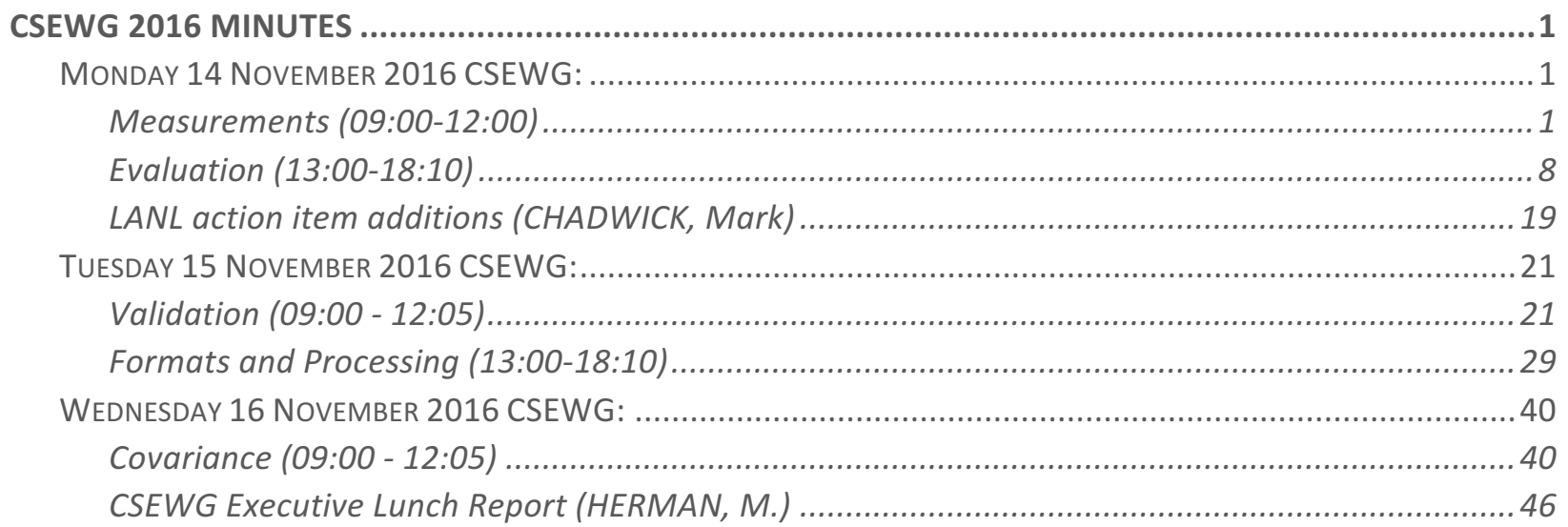

Legend:

Black: Comments

Red: Action items

Blue: Important note or question

Green: Resolved issue

Monday 14 November 2016 CSEWG:

Measurements (09:00-12:00)

- The progress at LANSCE experimental activity (LANL) LEE, Hye Young

O DANCE NEUANCE; neutron detector inside DANCE array; use as veto 
- $236 \mathrm{U}(\mathrm{n}, \mathrm{g})$ ratio to $235 \mathrm{U}(\mathrm{n}, \mathrm{g})$; systematic errors $\sim 4-8 \%$; good agreement with JEFF-3.1 ACTION: Should ENDF/BVIII be lowered? Also consider adjustment to the first resonance based on new $\mathrm{RPI}$ data

- 63Cu(n,g); makes 64Cu beta+ decayed 64Ni shields 64Zn in r-process; 25 keV MACS 97+/-4 mb ACTION: Compare with new ORNL+LANL evaluation in ENDF/B-VIII.beta3

- 239Pu(n,g) above 3×10^4 eV agrees with JENDL4; below in good agreement with ENDF/B-VII.1; no fission tagging; options to improve measurements using NEUANCE

○ Hellos \& Apollo @ANL

- $96 Z r(d, p)$ understand gamma str. ftn.

- $57 \mathrm{Fe}(\mathrm{d}, \mathrm{p})$

○ LENZ

- $160(n, a)$ in progress

- scheduled experiments: more (n,p), (n,a) up to $20 \mathrm{MeV}$ eg $\operatorname{Mo}(n, p), \operatorname{Cr}(n, a)$

- Work with Isotope Production Facility to produce radioactive targets; $56 \mathrm{Ni}(\mathrm{n}, \mathrm{p})$ planned, benchmark with stable $60-62 \mathrm{Ni}$

- Report on PFNS based on the ChiNu measurements (LANL) KELLY

$\bigcirc \quad$ ChiNu matrix $==$ nu(Ein)P(Eout $\mid$ Ein $)$

- $0.5-100 \mathrm{MeV}$ Ein

- PPAC fission detector

- Derive background from chance coincidence

○ 2 neutron detector arrays: low energy ( $<1 \mathrm{MeV})$ detector $226 \mathrm{Li}-$ glass, high energy (>0.5 MeV) 54 liquid scintillator; overlap of energy ranges 
O 235U; PFNS agrees well with ENDF/B-VII.1 \& III.Obeta; Low energy data higher than evaluation, likely needs further analysis; sample collection complete, must finalize analysis; complete 2017 \& publish

O 239Pu; preliminary; high event rate from decay alphas; 2.5 months with LE array, not HE array data yet; complete 2017

- Report on TPC and SPIDER progress (LANL) MAYOROV, Dmitriy

- SPIDER at Lujan; TKE \& TPC at WNR

○ SPIDER; measure FPY;

- 239Pu measured; SPIDER does differ from Lohengrin \& Cosi-Fan-Tutte measurements and England \& Rider; heavy peak shifted; ToF calibration for heavy fragment must be understood

- $235 \mathrm{U}$ measured with both arms

O TKE;

- TKE data in good agreement with Lestone for $238 \mathrm{U} \&$ 235U; Madland evaluation too smooth (misses 1st \& 2nd chance)

- Can also Mass yield as ftn of Ein; 3-5 amu resolution; 232Th data collected; $235 \mathrm{U}$ in progress

- NIFFTE fission TPC;

- 239Pu/235U fission cross section; agrees very well with ENDF/B-VII.1;

- angular anisotropy of fragments as well

- ORNL neutron cross section measurements for the NCSP (ORNL) GUBER, Klaus

○ GELINA @Geel (10 meV-20 MeV); total \& partial cross section measurements; user facility 
○ Old experiments CeO2; Enriched 142Ce oxide; transmission \& capture;

- DOE lease policy has very low activity requirement (0.6 $\mathrm{Bq} / \mathrm{g}$ ), in case of higher activation you have to buy; so had to run ORIGEN calculations to ensure can afford the experiment \& did test run at RPI with small sample;

- transmission \& capture data taken: serious discrepancies between data and evaluation; miss assigned \& missing resonances (WPEC-SG23); corrections won't be ready for ENDF/B-VIII; ORNL has plans to evaluate

○ 51V monoisotopic; $(n, g)$ missing resonances in ENDF; transmission, ENDF missing high energy resonances; data reduction in progress; won't be ready for ENDF/B-VIII; ORNL has plans to evaluate

- Experimental Activities in Berkeley (LBL) VOYLES, Andrew

O $(n, p)$ production cross sections

- use high flux neutron source $(\mathrm{d}-\mathrm{d}) 10^{\wedge} 8 \mathrm{n}$ 's $/ \mathrm{sec} / \mathrm{cm}^{\wedge} 2$

- Measured $64 \mathrm{Cu} ; 47 \mathrm{Sc}(n, p)$ cross sections by activation

- for medical isotope production.

- cross sections off by $2 x$ but tracked down to neutron energy spread \& kinematics; once corrected, in excellent agreement with ENDF, TALYS, other EXFOR; ACTION: LBNL compare to IRDFF evaluation

- stacked target charged particle excitation functions; preliminary results on several stacks of foils Ti(n,p), Cu(n,p)

o tunable neutron sources; $d$ from cyclotron breaks up on foil; high energy $\mathrm{n}$ beam; columnate; $1 \mathrm{MeV}-20 \mathrm{MeV}$

○ 56Fe structure; GRETINA@ANL (Leo Kirsch, his PhD thesis); Oslo reports increased gamma str ftn; $56 \mathrm{Fe}\left(p, p^{\prime} g\right)$ revised level scheme; measurements of lifetimes of many states (10-51 fs 
lifetimes); ACTION: If possible get level scheme to BNL; ACTION RESULT: Level scheme was transferred to BNL, there are 6 new levels around $6 \mathrm{MeV}$ that can't be included in ENDF evaluation, potential effect of the new levels on intensities of discrete gammas between low lying levels in $56 \mathrm{Fe}$ will be investigated.

- Recent Measurements on Carbon and Iron at the University of Kentucky Accelerator Lab (USNA) VANHOY, Jeffrey

O Nuclear structure with (n, n'g), monoenergetic beam; $\left(n, n^{\prime}\right)$ \& $\left(n, n^{\prime} g\right)$ angular distributions by moving detector carriage;

- Elastic \& inelastic cross sections with uncertainties typically $\sim 10 \%$

O 54Fe(n,el); paper heading to NPA < $31 \mathrm{Dec}$; data sent to S. Hlavac;

O 56Fe(n,el); paper to be submitted to PRC days; data sent to S. Hlavac;

○ *Fe(n,n'g); data taken;

- Angular distributions of for elastic scattering are different from ENDF/B-VII.1 below $2 \mathrm{MeV}$ incident neutron energy (back/forward ration seem lower then ENDF)

○ $12 \mathrm{C}(\mathrm{n}, \mathrm{el})$; initial measurement

- $12 C\left(n, n^{\prime} g\right)$; ran in Nov 2016, just before CSEWG; prelim. data in not terrible shape, but needs refinement

Other things want to try: $160(n, e l),\left(n, n^{\prime} g\right) ; 28 S i(n, e l),\left(n, n^{\prime} g\right)$; $24 \mathrm{Mg}(n, e l),\left(n, n^{\prime} g\right) ; 7 \mathrm{Li}(n, e l),\left(n, n^{\prime} g\right) ; 2 \mathrm{H}(n, e l)$

- Limits on $125 \mathrm{deg}$ excitation function... Characterize a4 coefficient in dsigma/dOmega gamma to pin down $2+->0$ transitions

- Nuclear Data Research at RPI (RPI) DANON, Yaron 
o 164Dy RRR; thermal total cross section agrees with ENDF; thermal capture RI 338+/-1 b ENDF 342.2 b; improvements to several resonances; doing SAMMY analysis

O $161,162,163,164 D y ;$ lots of new resonances \& some others that need to be removed

O Rh being analyzed, transmission, capture \& elastic small changes to thermal cross section compared to ENDF/B-VII.1

O $56 \mathrm{Fe}(\mathrm{n}, \mathrm{g}) 500 \mathrm{eV}-0.5 \mathrm{MeV}$; strong differences between JEFF3.1 \& ENDF/B-VII.1; ENDF/B-VII.1 has background that causes trouble with analysis, but isn't terrible;

- 96Mo RRR, data taken a few years ago, finally analyzing; will turn into revised RRR

- 56Fe scattering benchmark; measure Fe at several angles;

- beta2 very high 153 deg, $0.65 \mathrm{MeV}-0.85 \mathrm{MeV}$; beta1 close to data;

- beta2 low at 45 deg

- Uses FOM comparisons, but is hard to interpret; JENDL4 typically closer

- Beta2 (\& to lesser extent beta1) has somewhat wrong angular dependence; JENDL4 does better; seems that beta2 has systematically incorrect spin distributions leading to very different reconstructed angular distributions \& poor performance in these experiments

O ${ }^{*} \mathrm{Zr}$, < $0.5 \mathrm{MeV}$, preliminary, data shown; ENDF/B-VII.1 angular distribution OK; 45 deg shown

○ planned: scattering $\mathrm{Zr}$, $\mathrm{Hf}$ or $\mathrm{Cu}$; transmission $\mathrm{Hf}, \mathrm{Ta}$; photoneutrons; capture

- Current Status of the EXFOR Project (BNL) PRITYCHENKO, Boris 
- Compilations done once per paper, so not revisited for corrections unless is problem; need to let EXFOR folks know when there are corrections to be made

- NNDC responsible for US \& Canada experimental compilation; manages contract for S.Hlavac, O. Schwerer; collaborates with V.Zerkin

O Huge data sets ( $20 \mathrm{Mb}$ ) from ORNL crashed EXFOR editor, editor upgraded

- Troubles with OCR on really old papers with nasty tables

- New capability to upload experimental data to EXFOR webapp \& compare;

O REQUEST: would be interesting to make ratios of uploaded data with other data; ANSWER: such feature is already available

○ REQUEST: way to register to get notifications of uploaded/compiled data for say certain target/observable

- NIST Measurements and Standards, including Related Work at Other Facilities (NIST) CARLSON, Allan

- $1 \mathrm{H}(\mathrm{n}, \mathrm{n})$ angular distribution, done at Ohio $U$.

○ $6 \mathrm{Li}(\mathrm{n}, \mathrm{t})$ at NIST; work to reduce target mass uncertainty; IRSN mass off by $1 \%$; looking for funding to properly measure

○ NBS-I, US National fast-neutron source standard; calibrate vs. 252Cf; others

- $3 \mathrm{He}(n, p)$ coherent scattering length measurement in process

○ For new version of standards, including other measurements:

- $1 \mathrm{H}(\mathrm{n}, \mathrm{n})$ at Daub (U.Kentucky)

- $6 \mathrm{Li}(\mathrm{n}, \mathrm{t})$ Devlin (LANL), IRMM (Geel), Zhang (China); results in few percent diff above $1 \mathrm{MeV}$

- $10 B(n, a), 10 B(n, a 1 g)$; new standards 1-3\% lower 
- $C(n, n)$ Daub (U.Kentucky), RPI; revised fits not agreeing with these; Hale \& Paris eval driven by other data

- 197Au(n,g); Wallner (Vienna), Ullman (LANL); data agrees with evaluation well, so not much change

- $239 \mathrm{Pu}(\mathrm{n}, \mathrm{f})$ Tovesson \& Hill (LANL) agrees with current standards up to $10 \mathrm{MeV}$, then goes lower; accommodated in the ENDF/B-VIII evaluation

- $235,238 \mathrm{U}(\mathrm{n}, \mathrm{f})$ no new absolute data, just ratio data from n_TOF (CERN); agrees with evaluation very well

- $235 \mathrm{U}(\mathrm{n}, \mathrm{f})$ standard changes $>100 \mathrm{MeV}$ because of $239 \mathrm{Pu}$ data; is a big deal \& needs to be accommodated in the ENDF/B-VIII evaluation

- Thermal constants: MACS data not characterized well enough so only microscopic data used; resulted in changes from 2006 standards

- revised $233 \mathrm{U}(\mathrm{n}, \mathrm{f}),(\mathrm{n}, \mathrm{g})$, nubar,

- revised $235 \mathrm{U}(\mathrm{n}, \mathrm{f})$, nubar, agrees with beta3

- revised 239Pu(n,f), doesn't agree with beta3

- revised $242 \mathrm{Pu}(\mathrm{n}, \mathrm{f})$

- revised 252Cf

- REQUEST: method to disentangle which measurement(s) drive change to standards

\section{Evaluation (13:00-18:10)}

- Objectives - ENDF/B-VII beta 3 and next beta4 CHADWICK, Mark

- $235 \mathrm{U}, 238 \mathrm{U}$ changes are next big changes in beta4; accommodate standards changes

- beta5 only things that don't affect criticality 
- Paper writing must complete by summer

- Status of ENDF/B-VIII beta3 library - overview BROWN, David

- Timeline

- Beta0: thermal scattering, Decay, Cielo, non-Cielo

- Beta3: CIELO, non-CIELO

- U235,238, arrived late but should not change criticality

- Standards have not arrived yet

- ENDF Hackathon: Get together, finding and fixing bugs, review changes

- Reduction of $40 \%$ in open trackers, several old bugs were fixed

- Some big changes in Cu evaluations, U239 RRR, etc.

- Replaced SLBW by MLBW and corrected spins

- Some things could not be fixed in time: C12, Pu240, deuteron masses...

- Automated fixes will be implemented in the future

- Issue with Pu assemblies: attempt to fix with new Pu239,240

○ TODO: Add (n, $\left.n^{\prime}\right)$ gammas to isotopic C

- Tried to lower fission of Pu240 compared to VII.1

- Tweak of bound level of 240Pu to match Atlas values seems to bring performance close to VII.1

○ ACTION: (V. Sobes, D. Brown) ORNL refit 240Pu bound levels to match thermal fission as given in Atlas

- A summary of $8 \mathrm{~b} 3$ criticality performance v. 7.1 and $8 \mathrm{~b} 2$ KAHLER, Skip

- New CIELO files available at BNL \& IAEA

- Review of proper Doppler broadening processing controls in NJOY; NOTE: by default NJOY stops Doppler broadening at first 
threshold so it is every easy to get bitten by $56 \mathrm{Fe}, 235,238 \mathrm{U}$; a patch is in the works to make this much "safer"

- Fast assemblies: Overall performance same or slightly better than ENDF/B-VII.1 for all beta releases; beta4 candidate improves Flattop even more

- HMF007 w/ polyethylene comparable performance to VII.1

- HST still in excellent agreement even with standards based beta4 files

- PST has bias similar size to VII.1, but shifted down

- LCT unchanged

- Assemblies with steel or Fe, mixed bag of tests; beta3 systematically lower than beta2 results; beta2 on the whole does better than beta3; PMI2 still really bad, but less bad then in VII.1; LCT's (thermal lattices) slightly lower \& better with beta3 but essentially consistent

- PMF still lots of scatter; bare systems better but gets worse as add more reflector/moderators

- QUESTION: Can someone outside of LANL perform SINBAD, Reaction rates, and IRPhEP benchmarks?

- Current status of $235 U$ \& $238 U$ evaluated nuclear data files CAPOTE, Roberto

- 235U: ENDF/B-VIII.beta4=IAEA b25 (STD 2016)

- 238U: ENDF/B-VIII.beta4=IAEA b51 (STD 2016)

- 235U:

- TODO: RRR (n,g): Pigni < 100 eV, Leal 100 eV - 2.25 keV; refinement to eta/nubar; not merged in yet

- $\operatorname{RRR}(n, f):$ standards

- PFNS: Rising, Neudecker, GMA, GANDR all agree average energy is $2.00 \mathrm{MeV}$ 
- Nubar: add fluctuations caused by (n,gf); partly compensates change in leakage caused by PFNS change

- Fast $(n, g)$ : follows Jandel, Wallner \& Allmond data sets, helps with Na void reactivity problems noted by Leal \& JENDL4; cross section bounces around a little; Allmond data is $\operatorname{LLNL}(d, p)$ surrogate data but is only set in this energy but surrogate data has spin matching problems

- Fast $(n, f)$ : EMPIRE fission calculation tuned to match standards, but effects $\left.\left(n, n^{\prime}\right),(n, e)\right),(n, 2 n)$ and $(n, 3 n)$

- $14 \mathrm{MeV}$ neutron spectrum fixed in beta2 to match Kammerdiener, get very good pulsed sphere results

- 238U:

- preserve good $(n, f)$ below standards range

- $238 \mathrm{U}\left(\mathrm{n}, \mathrm{n}^{\prime}\right)$ reduced slightly $1 \mathrm{MeV}$

- $\left(n, n^{\prime}\right)$ feedback from CEA EXCALIBUR (Leconte \& Bernard (ND2016)); measured reaction rates in $238 \mathrm{U}$ cylinder irradiated by CALIBAN reactor

- $238 \mathrm{U}(\mathrm{n}, \mathrm{el})$ angular distribution

- agrees well with new TUNL (n,2n) data

- $238 \mathrm{U}$ pulsed sphere, beta1 not so good and B-VII.1 OK

- $14 \mathrm{MeV}$ neutron spectrum: do we match Baba or Kammerdiener? B-VII.1 matched Baba, but Baba has good shape but wrong norm. beta2=beta4 follows Kammerdiener and so matches pulsed sphere

- TODO: Standards not yet merged in

- Wallner AMS tightly constrains $238 U(n, g), 238 U(n, g) / 235 U(n, g)$ data, beta4 evaluations matches all

- 239Pu upgrades for 8 b3 CHADWICK, Mark 
- New standards increased $(n, f) \sim 0.5 \%$; managed to compensate with other improvements: nubar tweak removed, new PFNS, revised $(n, g)$ from DANCE

- QUESTION: BeRP ball (Snood \& Mattingly) have they tried out new evaluation?

- Below URR, in RRR, are much lower than standard; don't have time to re-evaluate RRR to resolve discrepancy

$\circ(n, g)$ modified

- nubar tweak removed; thermal nubar not yet integrated in; barely consistent

- TODO: beta4 will have $\mathbf{P}(\mathrm{nu})$; shouldn't affect criticality; uses format approved last year during CSEWG; gammas and neutrons

- TODO: fission gamma \& total gamma production files not included

○ TODO: FPY

○ TODO: covariances

- QUESTION: Ternary fission being accounted for in FPY evaluation? $d, t, 3 \mathrm{He}$, a production (gas production) from fission important, but small.

- RRR unchanged since WPEC SG

- Iron isotope advances NOBRE, Gustavo

- 56Fe heavy reliance on experimental data; IRDFF used where possible

- beta1, beta3: JENDL4 RRR, JENDL4 angular dist with tweaks to P2, P4; worse on benchmarks; better on RPI

- beta2: IRSN, reconstruct angular dist; better benchmarks; worse on RPI

- Rose inelastic shoulder by increasing gamma competition 
- Inelastic \& elastic angular distributions driven by data (Kinney \& Berthold)

- $54 \mathrm{Fe}, 57 \mathrm{Fe}, 58 \mathrm{Fe}$

- 54Fe capture matched to average cross section data from Allen; higher than RRR capture from IRSN; lower than beta1

- 54Fe total from IRSN higher than data from ORNL data; above 1.036 MeV EMPIRE average better than high IRSN

- Benchmarking beta3 close to beta1, mostly better than B-VII.1; beta2 different, also mostly better than B-VII.1; HMI problematic but can be changed with $54 \mathrm{Fe}$

- TODO: attempt revision of 56Fe RRR to remove Leal energy shift, attempt to fix spin assignments

○ TODO: HMS, inelastic gamma's

- TODO: FUND- VanDeGraff benchmark to investigate

- ACTION: Danon to plot beta3 against RPI capture data

- ACTION: try beta3 in RPI semi-integral experiment

- Copper ORNL resonance work SOBES, Vladimir

- New RRR

- ORELA transmission data 1 keV-300 keV

- GELINA capture 0.1 keV-300 keV (two sets, one corrected)

- $\mathrm{LRF}=7, \mathrm{Bc}=-1 ; \mathrm{MF} 32 \mathrm{w} / \mathrm{LCOMP}=2$

- RRR extended to $300 \mathrm{keV}$

- Matches Atlas D, R', thermal capture, RI; s-, p- capture widths got bigger

- Cumulative number of levels pretty good

- Merger with high energy data

- Fast matches smoothed resonances for all reactions 
- Angular distribution from reconstructed RRR, investigating merger with fast; doesn't match on very well

- Benchmarking

- IMI beta2 did OK, but beta3 systematically high \& depends on angular distribution

- HMF's of various kid, beta3 consistent

- Zeus beta3 better than B-VII.1, but still imperfect

- IMF all under predicting by a lot, but beta3 best

- TODO: compare to LANL data

- Livermore on $239 \mathrm{U}$ and 7Be \& charged particles THOMPSON, Ian

- 239U: added "fake" 2+ \& 3+ resonances on top of Brown-Trkov set

- 7Be: P. Page eval. stopped at $8.1 \mathrm{MeV}$; extended to $20 \mathrm{MeV}$ by gluing on TALYS 1.6 calculation

- ECPL elastic only $(p, t, h, a+a)$

- ECPL with transfer/breakup/charge exchange ( $p, d, t+7 L i, h+h)$

○ ENDL->GND->ENDF using FUDGE

○ IBANDL \& NIF both need CP data

O TODO: Hale \& co. do more comparison with ECPL data

- TODO: ECPL three-body out states cannot be put in ENDF currently awaiting LCT=4 Formats session discussion

- Thermal Scattering for heavy paraffinic materials MANRING, Cole

○ TSL for heavy paraffinic oil (liquid phase), used in systems as lubricant

- Used LAMMPS to model molecular dynamics of material

- TSL due to $n$ scattering off $H^{\prime}$ s in molecule

○ Have TSL cross sections 
- Light evaluations for C, $\mathbf{O}$ and the standards \& plans for b4 HALE, Gerry

○ 160:

- R-matrix analysis of $170^{*}$ system; $13 C(a, n)$ included in fit

- Elastic thermal scattering in B-VII.1 tuned to do well in benchmarks; CIELO value now much lower and on upper edge of what Kopecky \& Plompen want

- Lots of work sorting out normalization of (n,tot) data

- Unitarity locks down a lot of things...

- Checked against RPI total cross section measurement \& found good agreement

- 12C, 13C:

- lots of big renormalizations, confirmed by NIST

- tweaked $12 C(n, g)$ at high energy so makes more sense

- natC is $2 \%$ higher than VII.1; disagrees with standards

○ Light element standards $(1 \mathrm{H}, 6 \mathrm{Li}, 10 \mathrm{~B}, \mathrm{C})$ :

- extending n-p to $200 \mathrm{MeV}$;

- B standards evaluations not in agreement with one another

O Need relativistic R-matrix to put Hale \& Paris evaluations into ENDF format; also need Coulomb in \& out channels

- Notes on the consistency of $160(n, a)$ cross sections PIGNI, Marco

- ORNL evaluation based on evaluation by Sayer in 2000 using Reich-Moore approximation in SAMMY

- Bair (1973) vs. Harrisopulos dramatically disagree on $13 C(a, n) 160$

○ Corrected Macklin (1968) data matches Bair (1973) and West \& Sherwood (1982) 
○ Suggests much smaller renormalization for Harrisopulos (15\% vs. $42 \%)$

- Will make new evaluation with modified normalization

- A new cobalt-59 evaluation KAWANO, Toshihiko

○ Not appears to be important ;)

- Old evaluation from A. Smith (ANL)

O OK agreement with $(n, g)$ cross section data

$\circ(n, p) \&(n, n p)$ cross section in good agreement

O $(n, a) \&(n, n a)$ OK, but data is a-production cross section so must rework evaluation to compare

$O(n, 2 n)$ cross section good

$\circ\left(n, n^{\prime}\right)$ data strange shape for some higher levels, new evaluation good

- Gamma-ray production from $235,8 \mathrm{U}$ and $239 \mathrm{Pu}$ STETCU, lonel

○ For VII.1, PFGS given below $1.09 \mathrm{MeV}$, above only total gamma production cross section given

O In beta2, prompt fission gammas not added yet

○ Use CGMF (CGM=Monte-Carlo HF code; F=FF simulator)

○ 235U: follow Oberstedt $100 \mathrm{keV}-2 \mathrm{MeV}$; CGMF 0-100 keV, above $3 \mathrm{MeV}$; PFGS \& <Eg> \& <TKE>; Verbinski, Chyzh, Pleasonton

- 238U: similar approach as $235 \mathrm{U}$, but less data to compare against; PFGS \& <Eg $>\&<T K E>$; must revise spin to get $<$ Eg $>$ to increase

O 239Pu: compare to PFGS data from Chyzh

○ new file corrects issues in VII.1 \& beta2 where gamma data stuck in gamma production file

- FPY path forward for 8 CHADWICK, Mark SONZOGNI, Alejandro 
O IFPY vs. CFPY connected by decay data

○ Two independent evaluations (JEFF, ENDF/B)

○ GEF code

O Yields not compatible with decay data; example with $Z=44$ yields (ENDF vs. JEFF vs. GEF); example IRMM data for $238 \mathrm{U}$ appears to be off

○NDF format not flexible enough

- Impacts antineutrino yields, got basic science community spun up but appears to be artifact of bad data.

○ $96 \mathrm{Y}$ isomeric ratio fix based on measurement at ILL, new experiments planned

- 235U Resonance Parameters and Neutron Multiplicities in the Neutron Energy Region below $100 \mathrm{eV}$ and plans for ENDF/B-VIII.0beta4 PIGNI, Marco

○ VII.1 based on VI.8 from Leal, Derrien, Larson \& Wright (NSE)

- New evaluation consistent with neutron standards thermal values for fission, capture, elastic and eta \& alpha; these numbers consistent with CONRAD results from Noguerre

- Also matched average RRR cross section to averages in the standards evaluation

O Tweaks up and down RRR, valleys improved

○ Benchmarking, things got better (see Skip's talk)

○ Missing levels in 4- channel ( $p$-waves) above 500 eV, long term thing to work on

O TODO: include low energy part of RPI data and from n_TOF for fission \& capture by beta4

- NNL Thermal Scattering Law Evaluations for YH2 and Ice Ih HOLMES, Jesse \& ZERKLE, Michael 
○ $\mathrm{YH} 2(\mathrm{H} \& \mathrm{Y})$; High temperature moderator

- Keinert (1971) original "classical" model

- Redo using ab initio DFT calculation of phonon spectrum

- Match measured lattice parameter

- Phonon spectrum in reasonable agreement with Udovic

- Heat capacity in good agreement with experiment too

- Feed phonon spectrum into LEAPR module of NJOY

- 10 temps from room temp to 1000 deg. $\mathrm{K}$

- Validated with $\mathrm{H}$ scattering cross section measurements by Vorderwisch \& ... and Brand

O H2O hexagonal ice ( $\mathrm{H} \& \mathrm{O}$ ); criticality safety applications say when in transport \& storage of nuclear material

- Very different TSL behavior than liquid

- $\mathrm{O}$ in hexagonal lattice but molecules are actually disordered so have to capture global average structure

- Complicated phonon spectrum; different experiments validate different regions of phonon spectrum

- Inelastic scattering off ice has much more structure than liquid water

- Validate total cross section against L. Torres et al., NIMB 251 304-305 (2006)

- No criticality benchmark for ice, but is diffusion benchmark: Silver, NSE 34, 275-284 (1968); comparison quite well (time eigenvalue vs. buckling); not possible to manipulate regular $\mathrm{H} 2 \mathrm{O}$ to match benchmark.

- Words on big paper (s) CHADWICK, Mark

○ We are on the hook for it! Due May-ish, referee process over the summer. 
○ 100-150 pages: evaluations + validation

- CIELO papers in this issue, standards article and charged particle monitor paper in the issue too

- Jan 2018 issue

O TODO: Mark to send out format requirements both for text and for figures (using template generated by P. Talou)

○ Comprehensive paper, so need to be concise

○ When writing biblio, don't forget titles.

\section{LANL action item additions (CHADWICK, Mark)}

- Very highest priority:

- ACTION Brown/ORNL/Kahler/Trkov. Review proposed BNL $240 \mathrm{Pu}$ changes in the thermal and resonance region, which also adopts a previous developmental ORNL 240Pu resonance analysis, and (a) intercompare cross sections to assure we \& ORNL want to make this change, and (b) perform data testing with $\mathrm{Pu}$ assemblies involving significant 240Pu to check performance.

- ACTION Kahler - lots of data testing for beta4. (DItto for Trkov, VDM, White/Conlin ...)

- ACTION Chadwick/Talou/Kahler. After IAEA finalizes new standards, update 239pu to use standards and make other updates as needed to maintain criticality performance

- ACTION Hale/Kawano. 160(n,g) update above keV region for astrophysics

O ACTION Stetcu/Talou. Evaluate PFGS for u5,8 and pu9 for thermal and represent to $20 \mathrm{MeV}$, removing $1.09 \mathrm{MeV}$ format change. Chose evaluation that best represents data from Geel, Verbinsky etc, LANL based on view of data and theory. 
- ACTION White/Talou/Stetcu/Hale/Goorley. Create validation benchmarks for idealized scenarios of targets making gammas, comparing 7.1 and $8 \mathrm{~b} 4$ - to double check the evaluation hasn't lost key information on gamma production. Eg. 12C , 160 ...

- ACTION Kawano. 241Am thermal - update to new Harada/Jandel WPEC value. (also a small mod to $(n, 2 n)$ to reflect the shape of all $(n, 2 n)$ data including more recent data from TUNL, Geel, Athens).

○ ACTION Hale - any more work for standards project?

- ACTION Neudecker/Devlin. Usage of, and comparison to, final $235 \mathrm{U}$ chi nu in PFNS U5 evaluation.

○ ACTION Kiedrowski. Run beta4 against LLNL pulsed spheres.

- ACTION Kawano/Danon/Hale. Consider 9Be data and whether there is a basis to reduce keff calculated criticality of fast reflected systems by approx.. 200-500 pcm (though the crits. have significant discrepancies between them). VII.1 has a shift higher of about $500 \mathrm{pcm}$ versus VII.0 (owing to the higher total cross section from RPI). Scattering data, e.g. elastic, may point to less reflection to help this. RPI has semi-integral data - what do they suggest? Kawano/Danon/Hale will look at some scattering changes to see impact and study whether justifiable. Mark Cornock has commented on this too.

- Lower priority - but still important

- ACTION Tovesson/Talou/White. Compare TPC prelim data for Pu9(n,f)/U5(n,f) with new standards

- ACTION Devlin/Neudecker compare prelim chi-nu data for $239 p u$ with 8 b4.

- ACTION Goorley/Talou. gamma production benchmarks verification comparisons of $\mathbf{7 . 1}$ and $\mathbf{8 b 4}$ for NDSE scenarios. Extend to validation once validation data comes in. 
- ACTION Talou/Sood NCERC. BeRP ball sub-criticality performance and feedback on pu9 $8 \mathrm{~b} 4$ nubar etc.

- ACTION Kawano. Consider adopting lower $236 \mathrm{U}$ capture data using newest DANCE data

- ACTION Lee/Kawano. Soon send DANCE Cu63(n,g) from DANCE to Kawano/Sobes to compare with new Cu63.

- ACTION Kawano. Any changes needed to thermal Np237 ?

- ACTION Talou. Add in P(nu) and energy spectra (nu) for prompt neutrons and for prompt gammas, for U5,8 and Pu9

- ACTION Talou/Rising. Update to PFNS for minor actinides up to 5 MeV using Rising-Talou model?

○ ACTION Chadwick/Hale. Send Lisowski 6Li+t data to LLNL/Thompson

O QUESTION: UMF3,4 poorly perform - these have U3,8 in. Any changes in U3 that help? We have little time to do anything really ...

- QUESTION: Can NDAST/lan Hill point us to where to focus (in U3 say - probably no time to change U8).

- ACTION Kawano. Consider studying vanadium fast $>1 \mathrm{MeV}$ ? Angular distribution tweak to remove bias shape in Russian hmf25 trend with increasing $V$ reflector thickness.

Tuesday 15 November 2016 CSEWG:

Validation (09:00 - 12:05)

- BNL Advance System Update BROWN, David

O ENDF workflow: Evaluation->Phase 0 Tests->ENDF/A->Phase I testing (ADVANCE)->Feedback->Phasell testing (validation)

○ Phase 0: Rejected Automatically if simple tests fail 
- ADVANCE QA: Run battery of tests automatically for every commit

- Updates in ADVANCE: ACE tarballs; release note PDFs per isotope; upgraded fudge

- Example of build report from ADVANCE webpage

○ New version of INTER: Integral metrics, compare to other libs/exps, it is now part of FUDGE

O TODO: Add notifications to evaluators; new alpha sublibrary should be added; Fix ENDF manual; update NJOY;

○ Other desired upgrades: Add more codes; Covariance QA; Resonance and level scheme reports; Plotting more observables: nubar, PFNS, AD, energy spectra; Automated benchmarks

- New 2016 Integral Benchmark Data from ICSBEP and IRPhEP BESS, John

- Lots of slides mainly for reference on indico

○ Major international effort

- ICSBEP

- September 2016 release

- Shielding/alarm; Fundamental physics

- New versions of PU-MET-FAST-001 (Jezebel), ANL's ZPR$6 / 10, \ldots$ (Kurchatov), LCT071 (Valduc), Fund

- New additions: HMF083 asymetric to test early MC; HMF096 (SORA) pulsed with lots of Fe \& Be; LCT097 (SNL) with $\mathrm{Ti}$

- New ALARM-TRAN-CH2-SHIELD-001 (SILENE), ALARMTRAN-PB-SHIELD-001; FUND-NCERC-PU-HE3-MULT-002 (BeRP ball?)

O IRPhEP 
- Revised SCCA-SPACE-EXP-003; K worth not in good shape, not enough $\mathrm{K}$ in machine to pin down sensitivity

- Revised ZPPR-LMFR-EXP-011 (ANL ZPPR-2)

- Drafts: DIMPLE-LWR-EXP-003 (UK/CEA collab.); VENUSPWR-EXP-006 (Pu); DUKE-PWR-POWER-001 (Duke energy); MINERVE-FUND-RESR-001; IPEN(MB-01)-LWR-RESR-017 (reactivity vs. Temp); IPEN(MB-01)-....; LR(0)-VER-RESR-003 (Czech); RB-FUND-EXP-008 (Serbia); TRIGA-FUND-RESR002 (Slovenia) (rxn rate vs. position)

- Big collaboration to examine MA cross sections using Dounreay Prototype Fast Reactor (UK-Japan)

- Is there a benchmark sensitive to PFGS? No one could say for sure.

- IAEA CIELO Evaluations for 235,238U TRKOV, Andrej

○ Using benchmarks as guide to evaluation process

- EMPIRE calculations, but respect 2016 standards

○ Wallner \& Jandel experiments

- Standards caused big perturbation... so used DICE sensitivity profiles to guide what to tweak; e.g. lower $235 \mathrm{U}$ capture \& adopt IRMM U238 capture

○ Tweaked nubars

○ Pre-beta4 in pretty good shape in validation, but U233 not so good

- Data Testing at the IAEA TRKOV, Andrej

○ Scope of testing

- "Main" suite mostly in good shape; bare + reflected assemblies

- Looked at 2 sigma outliers in LANL 119 suite; hard to find trends even here 
O Discrepancies:

- $235 \mathrm{U}$ bare configurations have some inconsistencies:

Russian bare assemblies; ORNL sphere vs. cylinders; mass in Caliban

- Above thermal leakage fraction (ATLF)

○ Don't use FoM: "easy to game"

- E.g. r.m.s. $\Delta k$ or $\chi^{2} /$ d.o.f.

- one bad benchmark can mess up a FoM so bad whole library looks terrible

○ Processing code

- RR reconstruction, URR prob. tables, ...

- Cross check processing codes (PREPRO, NJOY, GRUCON)

- Reich-Moore formalism causes "anti-resonances" when hits zero width fission width; "fixable" using very small widths; makes unphysical "grass"; even $\Gamma_{\mathrm{f}}$ of $10^{-7}$ vs. $10^{-8}$ isn't enough, get wildly different unphysical results; Doppler broadening washes it out; not a problem with MLBW

- Probability Table Method (PTM); equivalent to PREPRO multi-band when derive bands from PTM; self-shielding is big deal

- LLNL ENDF/B-VIII.Obeta Data Testing JURGENSON, Eric

- Descalle's results from ND2016

- Several LLNL codes are going to use GND: Mercury \& GEANT using GIDI; FUDGE; GIDI

○ GIDI is API for GND

○ Suite of 84 tests in V\&V suite @LLNL

○ No TSL data, no PTM yet 
o Compare Mercury (w/ GIDI) vs. MCNP, they agree!

○ VII.1 vs. beta1 vs. beta2, all consistent with MCNP results

- Also have deterministic results using GID, but didn't get approved to show results in time

- ENDF/B-VIII.Obeta testing at AWE CORNOCK, Mark

○ Suite of spherically symmetric, all fast, U5/Pu/Mix metal

○ NJOY 2012.64

○ Deterministic tests

○ Processing errors

- FIX: 12C no MF1, MT451; FIX: Appears to be a local issue as file is present in beta3 evaluation

- 73As fixed in beta3

- FIX: 254m1Es \& 255Es no MF1, MT451; FIX: Appears to be a local issue as file is present in beta3 evaluation

- $56 F e$ fixed in beta1

- Use FoM's to summarize quality of simulations

- Mixed metal tend to be quite bad compared to VII.1; MMF7* series drives the disagreement; rest seem OK

○ HMF \& PMF look good

O QUESTION: Is Mix problems due to Be? All seem to have lots of Be in them; LANL testing showed mixed back (pardon pun), not clearly suggesting problem with Be; MMF7* series all Be reflected

Q QUESTION: Is there an issue with the spectrum used to weight the groups?

- PMF18 got worse, suggesting problem with Be or some other change

- Data Testing at ORNL WILLIAMS, Mark 
- APMX into CE \& MG libraries

○ Use CE-KENO, SCALE-6.2.1

○ "Valid Suite" chosen to span space of criticality conditions

o HST: usually really good except for HST14, they never work well; still a little low

○ LST: big experimental uncertainty, but overall really good agreement with beta3

- PST: lower than VII.1, better on average but some of the cases that worked before now are too low PST11's \& PST20's; Cd?

- Thermal LEU lattices somewhat better

- Mix lattices usually better, but MCT 4-001-4-011 got much worse

○ HMF same except HMF38's got better

○ PMF OK, some went down

○ IMF better on the whole

O HMF025-001 - HMF025-005, hasn't improved since VII.1;

- FKBN-2 experiments done in 2007 are Russian Federal Center Institute of Physics;

- HEU stacked disks; V reflected of various widths;

- Gets worse with more V;

- Sensitive to $235 \mathrm{U}$ (obviously) but also $V(n, t o t), V(n, e l) \&$ $V\left(n, n^{\prime}\right) ; V(n$, tot $) \& V(n, e l)$ is biggest contributor to change with reflector thickness;

- Can't tell if is angular distribution or total; angular distribution isn't part of sensitivity study so sensitivity might get pushed onto $\mathrm{V}(\mathrm{n}, \mathrm{tot})$;

- $\mathrm{V}$ is possibly NDAG action item

- Data Testing at CAB MARQUEZ DAMIAN, Jose Ignacio 
- Focus of group is on neutron sources \& advanced moderator development

O Contributed $\mathrm{H} 2 \mathrm{O}$ and D2O in collaboration with D. Roubtsov (CNL)

- Published results in EPJ w/ Noguerre, ANE and Nuovo Cimento

O D. Roubtsov found problem in heated $\mathrm{H} 2 \mathrm{O}$ in VII.1; resolved in beta3; experimentally validated, results submitted to EXFOR

- IPEN/MB-01 (Brazil), dos Santos, et al. NSE 133 (1999) and a few other publications; Tested using IRSN/CEA 235U, 238U \& IRSN 160; new TSL H2O dramatically improves; experiments sensitive to $235 \mathrm{U} \& \mathrm{TSL} \mathrm{H} 2 \mathrm{O}$, but not tested with ENDF

- Temperature interpolator for LEAPR module (Python scripts with XML intermediate files drive NJOY/LEAPR); QUESTION: is it possible to post interpolator on-line? QUESTION: is it possible to release LEAPR inputs for H2O? FIX: Yes, and though BNL in the not to distant future. NNL need them for licensing

- ACTION: BNL make system for storing LEAPR inputs associated with ENDF files

- Impact of Recent U.S. Nuclear Data on Graphite Reactor Benchmark Calculations BESS, John

- Available thermal spectrum graphite reactors: ASTRA, HTR-10 HTR-PROTEUS, VHTRC

O Prismatic HTGR

O HTR-10, HTR-PROTEUS: TRISO particles = UO2 wrapped in SiC \& PyC pebbles; in graphite block

○ Very sensitive to graphite TSL \& higher energy carbon

- Tested beta2 using natC \& 12C, 13C; isotopic C slightly higher but generally consistent

o HTTR not so good for data testing 
O HTR-PROTEUS shows modest improvement

- Analysis of a TREAT benchmark including graphite thermal scattering effects SORRELL, Nina

○ Nuclear grade graphite $\neq$ ideal graphite

- Nuclear grade graphite less dense, change to scattering is clear in experiment

○ Nuclear graphite in BNL-325? 1950's Kalefsky (spelling) transmission experiment

○ $10 \%$ porosity vs. $30 \%$ porosity vs. ideal

- Compared to ORELA experiment, looks good with $10 \%$ porosity

- Validate with TREAT reactor; relative activity vs. test pin location

$\bigcirc$ QUESTION: can we have a set of ENDF files with varying porosity?

- Data Testing with the PROTEUS Gas Cooled Reactor NEWMAN, Gareth

- PROTEUS reactor zero power reactor; lasted 40 years

○ 20 different core configurations ran from 1972-1979

- Modified core flux using variety of moderators and MOX fuel surrounding core where test objects loaded

○ Spectral indices: Foil activation \& fission chambers

- Gamma counting

$\circ$ Beta3 vs. VII.0 vs. VII.1; reaction rates beta3 agrees with VII.0 (the best) but slightly better than $(n, 2 n)$ reaction rate, but that one has huge errors so maybe we should discount as difficult to get enough MCNP statistics

- The Impact of Neutron and Photon Cross Section Libraries on ITER Neutronics Calculations BOHM, Tim

- Mohamed Sawan has retired

○ For fusion, radiation transport very important 
O Neutron fluence, gas production $(\mathrm{He}, \mathrm{t})$, radiation dose $(\mathrm{g} \& \mathrm{n})$

- FENDL library is collection of data deemed best for fusion applications, up to $150 \mathrm{MeV}$; FENDL-3.1 180 isotopes, most from VII.1 \& VII.0

- Photon heating is a big deal, but not a lot of effort put in to picking good evaluations

- Benchmark model, several concentric walls with vacuum vessel with plasma, then more walls in center

o In FENDL-3.1, 56Fe from JEFF-3.1.1, Cu from VII.1

- Photon flux very sensitive to neutron sublibrary as is nuclear heating

- mcnp84p vs. eprdata12; basically different versions of ENDF photonuclear sublibrary + photoatomic sublibrary

- Don't model charged particle reactions, ITER tells them the neutron flux from the plasma

○ TODO: MT205 gas production for W

- Skip is retiring and Andrej Trkov is now the new validation chair!

Formats and Processing (13:00-18:10)

- NOTE: ENDF format to freeze at mini-CSEWG, roughly June 2017

- Formats and related issues: Error in LAW=6 phase-space distributions HALE, Gerry

- N-Body phase phase formula makes sense in $\mathrm{CM}$ system

O $E^{*}$ not clearly explained in manual; Gerry has clarification for the formula for $\mathrm{E}^{*}$

○ APPROVED

○ ACTION: (D.Brown) Fix manual 
- C.Mattoon constructed a list of affected evaluations; ACTION: (D.Brown) make trackers

O $\mathrm{N}$ body phase space only used in three places:

- n-001_H_002, MT=16, used to describe the 2 outgoing neutrons +1 outgoing proton

- p-002_He_003, MT=111, used to describe 2 outgoing protons +1 outgoing deuteron

- d-001_H_003, MT=51, used to describe the outgoing $\mathrm{p}$ and $\mathrm{t}$ (after He4* breakup).

- The first one should be a good case to check how processing codes are currently handling phase space for the outgoing neutrons.

O The last one is a funny case: the reaction is $\mathrm{d}+\mathrm{H} 3->\mathrm{n}+(\mathrm{He} 4 *->$ $p+t)$. NBodyPhaseSpace in this case is only used for the final $p$ and $t$ (not the neutron), which seems to be an error (phase space is only meant to be used for 3 or more products).

- Ian is planning to replace that one with an ECPL evaluation with explicit pointwise distributions given for all three products.

- Formats and related issues: Need to define sign of spin in ENDF File 2 Manual MATTOON, Caleb

O In $\mathrm{LRF}=7$, sometimes $\mathrm{SCH}<0$, but it's a channel spin? Turns out ORNL was using it as the parity of the residual, but it's not documented in the manual.

- APPROVED

○ ACTION: (D.Brown) Fix manual "non-negative" not "positive"

○ ACTION: (C.Mattoon) fix Ca40, Cu63, Cu65 \& W183

- Formats and related issues: Add File 6 LCT=4 option: COM for initial products/Lab for decay products MATTOON, Caleb 
- Breakup reactions are ambiguous about what they mean by "center of mass" as there are several steps, each of which have their own center of mass

O Used in $\mathrm{d}+\mathrm{t}$ by $\mathrm{G}$. Hale \& will be used in the ECPL evaluations being translated by I. Thompson

○ APPROVED

○ ACTION: (D.Brown) Fix manual

○ ACTION: (LLNL) Finish translations

- Formats and related issues: Floating point formats ROMANO, Paul

O FIX: Adjust wording E is optional, dropping it gets you 1 digit of accuracy

○ FIX: Change letter " $e$ " to something else, maybe " $m$ "

○ FIX: "Whitespace" to "space character"

- APPROVED

- ACTION: (D.Brown) make fixes and update manual

- Clarify masses and $\mathbf{Q}$ values $B E C K$, Bret et al

- Masses in ENDF do not always agree with Q's given in ENDF, sometimes off by many electron masses

○ ENDF rules not followed

O Recommend for ENDF:

- Keep rule 0.5.1.1 on atomic and nuclear masses

- Change rule 3.1.1: Q value rule to be atomic masses rather than nuclear masses, even though they are not given in the ENDF files

- In FUDGE/ADVANCE: check Q's masses, with tolerance less than the electron mass (0.1 MeV?)

○ APPROVED 
○ ACTION: (D.Brown) Fix manual

○ ACTION: (LLNL) Update FUDGE behavior for change

- ACTION: (BNL) Update NNDC checking codes to respect change

○ Recommend for GND:

- Use atomic masses everywhere,

- Assume "electron always going with a proton", so not $p$, but $1 \mathrm{H}$

- ZAID \& MAT clarifications for TSL data DAVID, Brown

- MAT number assignments approved

○ APPROVED

○ ACTION: (D.Brown) Make changes in manual, circulate assignments to TSL evaluators

○ ZAID clarified: Proposed convention for TSL evaluations (Rule 2 in Appendix C)

- 2.1) TSL associated with particular nuclide, the nuclide ZAID should be used;

- 2.2) If TSL is associated with an element, it should use the ZAID of the element;

- 2.3) TSL for compound or mixed moderator. After some discussion, description in Appendix $\mathrm{C}$ was updated to keep this option only for historical compounds with note that this option is not recommended for future TSL evaluations.

○ APPROVED

O ACTION: (D.Brown) Make changes to manual, but use ZA, not ZAID

- MT458 Fission energy Release WHITE, Morgan

○ Fission energy release format is given as a polynomial 
- Measured energy release doesn't look very much like a (low order) polynomial; a TAB1 would be safer

- A TAB1 would allow more accurate connection between energy release and other energy balance calculations

- Allows covariance using MF31/33 like arrangement

- Issues to consider in drafting full proposal

- Covariance

- Procedures

- $\mathrm{FCl}$ table

- Consistency between other files and this one

- Old vs. n ew format for sketchy vs. detailed numbers

ACTION: (M. White) Flush out and make full proposal before the mini-CSEWG meeting

O ACTION: (M. White) Send the 3 new LANL evaluations with fission energy release data in the new proposed format to processing code developers along with NJOY-processed results in order to provide verification for implementation in FUDGE and AMPX.

- BNL Activities Related to Formats and Processing and ENDF-102 Manual Updates BROWN, David

- Updated version of ENDF manual will be made available

○ Revised ENDF manual should be frozen having in mind the VIII.0 release approaching

- 6 Error/typos already fixed

○ Constants in appendix $\mathrm{H}$ were updated

- ACTION: (Volunteer with sufficient gravitas) New preface needed for the manual

○ ACTION: (D. Brown) formats-chair fix 
- Cycle of a format change within GForge

- ACTION (A. Trkov/R. Capote): Log normal flag for covariance: tracker (ID 900) is open for long time waiting for an example file. Keeping open until mini-CSEWG

O ACCEPTED: Allowing MF=4/5 for incident gamma evaluation.

- ACTION (D. Brown) Wording about this in the manual should be rephrased for approval at mini-CSEWG

- Other items for proposed changes have already been approved in earlier talks

- ACTION: (D.Brown) recirculate punchcard description and kinematics discussions

- Status Report on WPEC SG38 Format Development BECK, Bret et al.

O New format to bring modern tools, replacing ENDF-6

- WPEC Subgroup was created with the original intent of replacing ENDL

- Subtasks were defined, some have been completed while others will continue under SG43 (and SG-B)

- Will the publication of 4 "requirements and specifications" documents (basic data containers, ...) SG38 will be completed

- Basic data containers: 1-d functions (cross sections, ...), changing of interpolations regions, 2-d functions (i.e. Angular Dist.)

- Organization of data: GND reactionSuite, containing list of all reactions. Each reaction stores a cross section and output channel with $Q$ value and list of products

○ Style section can store different types of data

- New subgroups will officially start in May 2017

- Sg-38 produced a format specification and should now focus on disseminating the format and building infrastructure 
- SG-B will take on format governance while SG-43 will expand the infrastructure for generating checking manipulating and using GND-formatted data

- Processing code reports: NJOY KAHLER, Skip

○ NJOY has been around for $\sim 40$ years; most widely used version was NJOY99; current version is NJOY2012

○ NJOY2012

- Unofficial updates available at NEA databank

- Gotta pay LANL if you want it

- Current latest patch is up50

- New patches are nearly complete, will go with NJOY2016; available upon request

○ NJOY2016

- ND2016 will be open source (BSD!);

- working on manual, it is a big job;

- will use new distribution model

- NJOY Training available; see MCNP training page; advertised in RSICC newsletter as well

○ NJOY21

- Ground up rewrite of NJOY

- Backwards compatible with NJOY2016

- Use both ENDF \& GND; improved diagnostics; faster; easier to maintain

- Plan: wrap legacy with C++; replace functionality as go along; test to make sure didn't break anything

- Test driven development (unit, system, integration), see http://travis-ci.org/njoy/ 
- Modules: utility, math, nuclearData (classes), ENDFtk (ENDF I/O), ACEtk (ACE I/O)

- See http://njoy.lanl.gov, http://github.com/njoy

- Open source, no export controls

- Processing code reports: AMPX WIARDA, Dorothea

- Updates to API classes;

- ENDF resonance parameter classes and covariance matrix API

- API has essentially getCrossSection() and getAngularDistribution() functions

- Currently only RM implemented in C++, rest still use old code

- Derivatives from SAMRML

- Group averaged derivatives function, used by PUFF, with sandwich formula get covariance

- API also has average level spacing and width functions

- Gridding setup to be $0.1 *$ sqrt(Gg/D)

- In memory resource for COVERX, C++ with fortran bindings; covariance I/O; convert between rel \& abs cov.

○ Updated PUFF; new user input; better error reporting; use new API's (COVERX, ENDF and resonance); more testing; crossmaterial covariances; PUFF understands GND covariances

- COVCOMP, a COVERX comparison program; can be called standalone or with API

- Cognac, covariance correction code

○ POLIDENT, handles pointwise OK data, uses new API

O Issues:

- Numerical instability in Coulomb function causes trouble with new Ca40 evaluation; impacts POLIDENT, SAMRML \& 
SAMMY; Use new code described in N. Michel, CPC 176, 232-249 (2007)

- In " $\mathrm{H}$ in $\mathrm{H} 2 \mathrm{O}$ ", the range extended to $10 \mathrm{eV}$; $\mathrm{Y} 12$ updated to use short collision time approximation if any values interpolate to 0 ;

- Found many issues in beta2, 3, since fixed

O Available in SCALE 6.2, precompiled AMPX in 6.2.1.; AMPX manual not export controlled, available on SCALE website (http://scale.ornl.gov/)

- Processing code reports: FUDGE BECK, Bret

O "protare": PROjectile + TARget + Evaluation; for example " $n$ ", "Fe56", "ENDF/B-VIII.0"

- GND: multiple processed data can be stored along with evaluation in file, denote with "style"

○ FUDGE:

- "arbitrary" manipulation of data

- $\mathrm{C} / \mathrm{C}++$ for tough calculations

- Multi-threading for RR \& MG (not heating yet)

- open source on NNDC GForge website

- processing (processProtare.py script)

- resonance reconstruction

- convertUnits(\{'eV':'MeV', ...\})

- average product data

- heating

- cdf's from pdf's

- $M G$, including upscattering 
- TODO: URR, multiband, TSL (w/ LEAPR), kiwi (covariance realizations), GNDtoACE, documentation

○ GIDI: discussed in C. Mattoon \& E. Jurgenson's talks;

- testing in ARDRA, in Mercury already

- needs on-the-fly multigrouping

- angle biasing

- faster sampling

○ Python 2 end-of-life is 2020, so we need to port to 3

○ Future work/capabilities needed in FUDGE

- URR probability table generation

- Multi-band

- Thermal scattering

- Thermal upscatter for deterministic transport

- Kiwi package that uses FUDGE to generate realizations of nuclear data from mean and covariance data

- Fix GND to ACE and maybe others

- Documentation

- Status of TSL Code Development at NCSU ZHU, Yuwei

O Modular code in F95, some C++; Dynamic memory; wrapped in Python; GUI with QT

○ Hopefully available through RSICC

○ No short collision time approximation

- Has coherent inelastic, exact coherent elastic, OpenMP, GUI

- You give it a phonon spectrum, it makes plots \& ENDF files

o Compares well to THERMR+LEAPR

- Coherent inelastic compares well with data 
○ Coherent elastic

- Relaxes several approximation

- No hard-wired stoichiometry (such as in NJOY)

- SAMMY Modernization ARBANAS, Goran

O Overview of SAMMY 8.1: Few bugs fixed, beta release to follow

O Overview of API design for SAMMY modernization

○ New features of 8.1: Integrated benchmark exp. Information, developed under SCALE SQA like AMPX (automated CMake/CTest suite, revision control), updated physical constants, corrected index leading to incorrect matrix multiplication

- New version: SAMSON

○ API diagram defined before implementation

O Experimental Effects: Doppler broadening, Neutron transport, resolution function

O SHIFT: neutron transport code, would enable fitting integral benchmark experiments; MPI enabled; possibly could use MCNP input

- In principle entire experimental setup could be simulated: Fitting to raw data may or may not be possible

○ Fitting API: Generalized Least Squares (GLS), Total Monte-Carlo (more realistic uncertainties, fit differential and integral benchmark exp. data

- Recently a preliminary interface was discussed

○ Use of Froehner's formulation and notation for GLS fit

O C++-array library being considered: Parallelized via BLAS library

- Can handle main ENDF resonance options

○ Will use Mesh Generation 
O Recent developments: Generalized Reich-Moore approx. (presented at ND2016), converted resonance parameters to formal R-matrix, S-matrix pole representation of R-matrix (useful for on-the-fly Doppler broadening); Sammy computes only open channels, effects of closed channels being investigated

Wednesday 16 November 2016 CSEWG:

\section{Covariance (09:00 - 12:05)}

A meeting of the CSEWG Covariance Committee that lasted approximately $2 \frac{1}{2}$ hours was held during the 2016 annual CSEWG meeting that, itself, was a component of Nuclear Data Week 2016. The covariance meeting was comprised of a single session that took place on Wednesday morning, 16 November. This meeting, by design, consisted mainly of informal discussions on the status of covariance data to be considered for inclusion in ENDF/B-VIII.0, as well as on more general technical problems that are being encountered in covariance evaluation. There was also an informal presentation by Marco Pigni that described an ORNL initiative to develop improved evaluation methods for fission-product yield data. Dave Brown gathered and organized information extracted from the GFORGE repository on the status of covariance data already submitted, pending, or likely to not be available in time for the forthcoming release of the next ENDF library. This material formed the basis for structuring the meeting discussions. In addition, Caleb Mattoon utilized the same database to generate plots with NJOY that could be used when discussing issues related to specific materials and reaction processes. Finally, Gustavo Nobre took comprehensive notes during this meeting. All of these material will be made available, along with the present summary, as a record of this meeting.

It was noted that the long-standing problem of too-low cross-section uncertainties in the low-energy region, mainly due to neglect of correlations, persisted in several instances, and that excessively low predicted uncertainties are more damaging from the perspective of users than too- 
large uncertainties because they give the false impression that the state of affairs is better than it is in reality. It is unlikely that this issue can be addressed for ENDF/B-VIII.O in all instances where it is observed to occur, but it was agreed that an effort should be made by evaluators, at least for the most important materials, such as U-235,238, Pu-239, and Fe-56, to find a physically justifiable ' $\mathrm{fix}^{\prime}$ ' to this problem prior to inclusion of these covariance data in the library. Such a 'fix' (or 'fixes') should certainly be clearly documented in the Nuclear Data Sheets ENDF/B-VIII.O 'Big Paper'.

It was further agreed that high-quality standards that have been established for the ENDF general purpose library covariances should continue to be adhered to for ENDF/B-VIII.0, even if it means leaving gaps in the availability of covariances for some materials and processes, rather than trying to augment this library with 'low-fidelity' covariance information or ad hoc covariances unrelated to the evaluation processes that generated corresponding central values. Users commented that they routinely supplement their user-based data libraries with information from other sources when such information is needed but not available directly in the ENDF library. Therefore, it was concluded that there is no pressing justification for CSEWG to consider lowering its quality standards just to satisfy specific needs for various special applications.

A time roadmap was established during the meeting for submitting and testing covariance data. It was agreed that late March 2017 would be the cutoff time for accepting submitted covariance information that is not yet available. Phase 0 and 1 machine testing of submitted files would be accomplished at the NNDC at the time of submission (ADVANCE). During April and May, Phase 2 testing, including processing-readiness, of these files, will likely be performed by ORNL and LLNL. Other volunteers may also inspect these data visually for problems that cannot be easily spotted by machine inspection. The goal is to complete the covariance QA and acceptance process prior to the mini-CSEWG meeting that will most likely take place in May. The final release of ENDF/B-VIII.0 will probably be in late summer of 2017. 
- Introduction SMITH, Don

- Jan 2017-ish, mean values due in beta4

o Late-Feb 2017, get $1^{\text {st }}$ pass at covariance checks

- Start TSUMANI \& FUDGE checking of covariances in Mar-Apr

- Freeze for mini-CSEWG

- Review of covariance inventory BROWN, David

○ Neutron on neutron: low priority

○ ACTION: (G. Hale) 12C,13C

- 40Ar: No covariance data are coming

- 160:

- covariance model exists but needs to be updated

- ACTION: (G. Hale) 160 (CIELO)

- ACTION: (G. Hale) 1H (CIELO), with special focus on angular distributions

- 14N: No, would need to redo evaluation

○ Manufacturing covariances?

- Investigating using TENDL for low-fidelity covariances

- If covariances are not produced with evaluation they should not simply be filled without deeper investigation

- Cross-reaction and cross-material covariance are not available, would be a big project by itself

- Covariances calculated separately using different methods for fast and resonance region present problems if not combined properly. Deadline of end of March to determine proper way of establishing correlation between the different regions should be ok. 
- M. White strongly objects putting anything other than covariances done in the proper way. He advocates that it is better to have nothing than something that is not completely reliable.

- Having too-small uncertainties is much worse than having overestimated uncertainties.

- $\mathrm{Mn}$ is an example on how to extrapolate uncertainties from Fast to Resonance regions to do a proper correlated merge. This works for total and elastic but still has problems for capture. Think of a (simple) solution for correlated covariance for capture (Extending EMPIRE down?)

○ Covariance testing/benchmarking

- ORNL needs 2-3 months

- LLNL can test too?

o Iron:

- will have covariances (previous release already had preliminary covariances)

- ACTION: (BNL) * Fe covariance (CIELO)

- Cobalt, Nickel: No covariance data are coming

- Arsenic, Krypton, Rh, Te: No covariance data are coming

○ Hf: No covariance data are coming

o Pu239:

- missing covariance data of all kinds.

- For $\mathrm{P}(\mathrm{nu})$ covariance, data exists but would need ENDF format change and there isn't time for it

- For VII.1 Pu Luiz converted file 32 to group structure for file 33 
- ACTION: (P. Talou) Ready by end of February: Cross section and nubar covariances.

- ACTION: (D. Neudecker) Ready by end of February: PFNS

- ACTION: (M. Pigni) Resonance covariances

○ U235:

- ACTION: (M. Pigni) generate for resonance paramenters

- ACTION: (R. Capote and A. Trkov) No new fit of nubar for U235 was done. IAEA will figure out what to do.

- U235 VIII.0beta3 contains covariances for MT=18,102. Uncertainties are small but that makes sense (Caleb's plots). In capture there's a jump to $30 \%$ uncertainty around $1 \mathrm{keV}$

○ U238:

- ACTION: (M. Pigni) Use old covariances and repair them

- ACTION: (M. Pigni) will assess the situation with the Big 3 and report what will be needed

○ U235,238: mean values should be done before the end of January. Tentative timeframe for covariances would be end of March

O ACTION: (M. Dunn) send ORNL group structure to R. Capote

- Problems with processing of $L R F=7 \mathrm{LCOMP}=2$ covariances with NJOY

- Cu has LRF=7 covariances

- ACTION: (Kahler) investigate processing problem in NJOY2012

- ACTION (C. Mattoon, D. Brown) Integrate the covariance plotting to ADVANCE

- Situation of Covariances for Standards: 
- Were not submitted yet (mean values haven't been submitted either). Cross-reaction were missing in VII.1, while JENDL has it and has even cross-isotope correlation. For standards uncertainties/covariances will come out together with the mean values. They tend to be on the lower side.

- ACTION: Standards will be submitted to BNL by December 15

- ACTION (C. Mattoon, D. Brown) Make Caleb's report (as is) available online

○ $U$ \& Pu will have the issue of too low uncertainty due to lack of cross correlation resolved

$\circ \mathrm{U}, \mathrm{Pu}, \mathrm{Fe}, \mathrm{O}, \mathrm{C}$ will have these covariance issues related to crosscorrelation addressed

- Fission Product Yield work at ORNL PIGNI, M.

- Propagation of fission products uncertainties from VII.1 led to too big uncertainties

○ New funding for fission products covariances

○ M. Pigni wrote code to calculate correlation for fission product yields

- Tool is needed for full evaluation of fission products yields, from physical model to uncertainties and correlation. Collaboration with T. Kawano.

- Goal is to complete a covariance library. No time for VIII.0, but for future

- Possibility of storing a relative covariance library for cases where the mean values are not changed

- Importance of consistency between independent and cumulative yields 


\section{CSEWG Executive Lunch Report (HERMAN, M.)}

- NDS special issue January 2018:

O Big paper on ENDF/B-VIII.0, evaluation and validation in the same paper, M. Chadwick is leading author

○ Set of CIELO papers:

- M. Chadwick - overview

- Plompen - 016

- Herman - Fe

- Capote - U235,2388

- Neudecker - PFNS evaluation for U, Pu

- Devlin - PFNS experiments and Chi-nu

- Mosby - Pu capture experiments

- CEA fast evaluations (not committed)?

- Paper on neutron standards, lead author is A. Carlson

O Paper on monitor reactions for charged particle induced reactions (IAEA CRP), lead author is A. Hermanne

- Beta4: January 30, last unrestricted beta release

- Beta5: May-June, possibly correlated with mini-CSEWG (no changes affecting validation!!)

- Beta6: 2 weeks before final release. Should be equal to Beta5. It will essentially be VIII.0 version. Catch typos, etc..

- Release - late summer

- Andrej Trkov replaces Skip Kahler as chairman of the validation committee

- Alejandro Sonzogni replaces M. Herman as chairman of the CSEWG

- Next CSEWG: BNL - November 6-9, 2017 
